\title{
VECTOR FIELDS ORTHOGONAL TO A NONVANISHING INFINITESIMAL ISOMETRY
}

\author{
CHAO-CHU LIANG
}

\begin{abstract}
Let $X$ be a nonvanishing infinitesimal isometry on a compact Riemannian manifold $M$. If there exists a nonvanishing vector field orthogonal to $X$ and commuting with $X$, then the Euler characteristic of the complex consisting of all the differential forms $u$ satisfying $i(X) u=0=$ $L(x) u$ is zero.
\end{abstract}

Let $X$ denote a nonvanishing infinitesimal isometry on a compact Riemannian manifold $M^{n}$. Let $A(M)=\left\{A^{k}(M), d\right\}_{0<k<n}$ denote the de Rham complex of $M$. We let $i(X)$ denote the interior product operator, and $L(X)$ the Lie derivative on the elements of $A(M)$. We define $E(M)=\{u \in$ $A(M) \mid i(X) u=0=L(X) u\}$. The cohomology of the complex $\{E(M), d\}$ can be considered as the cohomology of the "orbit space" $M / X$ [5]. Let $\langle$, denote the metric on $M$. The purpose of this short note is to prove the following.

THEOREM. If there exists another nonvanishing vector field $Y$ on $M$ such that $\{X, Y\}$ forms a set of linearly independent, commuting (i.e., $L(X) Y=[X, Y]$ $=0)$ vector fields on $M$, and $\langle X, Y\rangle=0$, then

$$
\chi(M / X)=\sum_{i=0}^{n-1}(-1)^{i} H^{i}(E(M))=0 .
$$

Proof. According to [4, p. 535], $X$ gives rise to a 1-dimensional foliation with bundle-like metric, and a differential form $u$ is base-like in the sense of [4, p. 531] if and only if $u \in E(M)$.

Let $w$ be the 1 -form defined by $w(Z)=\langle X, X\rangle^{-1}\langle X, Z\rangle$. It was shown in [3, pp. 188-189] that $d w \in E^{2}(M)$, and $H^{*}(E(M))$ is finite dimensional. Hence $\chi(M / X)$ is an integer.

If $\bar{*}: A^{P}(M) \rightarrow A^{n-P}(M)$ is the usual Hodge star operator, then there exists a star operator $*: E^{p}(M) \rightarrow E^{n-p-1}(M)$ defined by ${ }^{*} u=i(X /|X|)^{\bar{*}} u$ [4, p. 532]. As in [4], we define $d^{\prime}=(-1)^{(n-1)(p+1)+1 *} d^{*}$.

For $a, b \in E^{p}(M)$, we define the inner product by the formula

Received by the editors September 5, 1978.

AMS (MOS) subject classifications (1970). Primary 57D25, 58G99.

Key words and phrases. Infinitesimal isometry, vector fields, transversally elliptic operators, Euler characteristic.

'Supported by the University of Kansas General Research Fund. 


$$
(a, b)=\int_{M} a \wedge^{*} b \wedge w
$$

Let $\varphi \in E^{p}(M)$ and $\psi \in E^{p+1}(M)$, then we have

$$
\begin{aligned}
0 & =\int_{M} d\left(\phi \wedge^{*} \psi \wedge w\right) \\
& =\int_{M} d\left(\phi \wedge^{*} \psi\right) \wedge w+(-1)^{n-1} \int_{M} \phi \wedge^{*} \psi \wedge d w .
\end{aligned}
$$

Since $d w \in E(M), \varphi \wedge^{*} \psi \wedge d w \in E^{n}(M)=0$. Hence, we have

$$
\begin{aligned}
0 & =\int_{M} d\left(\phi \wedge^{*} \psi\right) \wedge w \\
& =\int_{M} d \phi \wedge^{*} \psi \wedge w-\int_{M} \phi \wedge^{*} d^{\prime} \psi \wedge w=(d \phi, \psi)-\left(\phi, d^{\prime} \psi\right)
\end{aligned}
$$

Therefore, $d^{\prime}: E^{p+1}(M) \rightarrow E^{p}(M)$ is an adjoint of $d: E^{p}(M) \rightarrow E^{p+1}(M)$ with respect to the above inner product. (REMARK. The referee has pointed out to us that Reinhart's construction of an adjoint [4, pp. 532-533] is incorrect. The main problem is that the expression $\phi \wedge \wedge^{* \prime} \psi \wedge d x^{1}$ $\wedge \cdots \wedge d x^{p}$ on p. 533 depends upon the choice of coordinates, and usual integration by parts construction of an adjoint does not go through.)

The rest of Reinhart's proof can be carried through here (Laplacian, Green's operator, etc.). As in the proof of the classical Hodge theorem, it follows from Reinhart's theorem that the index of the operator $D=d+d^{\prime}$ : $E^{\text {even }} \rightarrow E^{\text {odd }}$ is $\chi(M / X)$, where

$$
E^{\text {even }}=\underset{p \text { even }}{\bigoplus} E^{p}(M) \quad \text { and } \quad E^{\text {odd }}=\underset{p \text { odd }}{\bigoplus} E^{p}(M) .
$$

As in [2, p. 87], we note that the operator $D$ is not elliptic, but only transversally elliptic.

If there exists a nonvanishing vector field $Y$ satisfying the condition of the theorem, then we may regard $Y$ as a 1 -form in $E(M)$ by using the Riemannian metric on $M$. Following [1], we may consider the operator $R(Y)$ on $E(M)$ given by the right Clifford multiplication of $Y$, and $R(Y)$ is an automorphism of $E(M)$ (at each point $x \in M, E(M)_{x}$ is an exterior algebra of dimension $\left.2^{n-1}\right)[1$, p. 10]. As in [1], we see that the operator $(d+$ $\left.d^{\prime}\right) \circ R(Y)-R(Y) \circ\left(d+d^{\prime}\right)$ is of order zero. Let $D^{\prime}: E^{\text {odd }} \rightarrow E^{\text {even }}$ denote the adjoint of $D$. Then the argument in $[1$, p. 11] showed that index $D=$ index $D^{\prime}=-$ index $D$. Hence $\chi(M / X)=$ index $D=0$. Q.E.D.

Corollary. Let $M^{2 k+1}$ be a compact Riemannian manifold having the (real) cohomology of a $(2 k+1)$-dimensional sphere. If $Y$ is a vector field commuting with a nonvanishing infinitesimal isometry $X$ on $M$, and $\langle X, Y\rangle=$ 0. Then $\{X, Y\}$ must be linearly dependent at some point of $M$. 
Proof. According to [3, pp. 188-189], the cohomology $H^{*}(E(M))$ is the same as the cohomology of the $k$-dimensional complex projective space $C P(k)$. Hence the Euler characteristic $\chi(M / X)=k+1$. Q.E.D.

\section{REFERENCES}

1. M. F. Atiyah, Vector fields on manifolds, Arbeitsgemeinschaft für Forschung des Landes Nordrhein-Westfalen, Heft 200, Westdeutscher Verlag, Cologne, 1970.

2. __ Elliptic operators and compact groups, Lecture Notes in Math., vol. 401, SpringerVerlag, Berlin-Heidelberg-New York, 1974.

3. W. Greub, S. Halperin and R. Vanstone, Connections, curvature, and cohomology. Vol. II, Academic Press, New York-London, 1973.

4. B. L. Reinhart, Harmonic integrals on foliated manifolds, Amer. J. Math. 81 (1959), 529-536.

5. G. W. Schwarz, On the de Rham cohomology of the leaf space of a foliation, Topology 13 (1974), 185-187.

Department of MATHEMATICS, University OF KANSAS, LAWREnCE, KANSAS 66045 